\title{
Number of maximal partial clones
}

\author{
Karsten Schölzel \\ Institut für Mathematik \\ Universität Rostock \\ Ulmenstraße 69 \\ 18057 Rostock, Germany \\ Email: karsten.schoelzel@uni-rostock.de
}

\begin{abstract}
All maximal partial clones on 4-element, 5-element, and 6-element sets have been found and are compared to the case of maximal clones of all total functions. Due to the large numbers of maximal partial clones other criteria to check for generating systems of all partial functions are analyzed.
\end{abstract}

\section{Introduction}

In many-valued logic finite basic sets are considered. We only have to consider the set $E_{k}:=\{0,1, \ldots, k-1\}$ with $k \geq 3$ being fixed in the rest of this paper.

The set $P_{k}:=\left\{f^{(n)} \mid f^{(n)}: E_{k}^{n} \rightarrow E_{k}, n \geq 1\right\}$ is the set of all total functions on $E_{k}$. Let $D \subseteq E_{k}^{n}, n \geq 1$ and $f^{(n)}: D \rightarrow E_{k}$. Then $f$ is called an $n$-ary partial function on $E_{k}$ with domain $D$. We also write $\operatorname{dom}(f)=D$. Let $\widetilde{P}_{k}^{(n)}$ be the set of all $n$-ary partial functions on $E_{k}$ and

$$
\widetilde{P}_{k}:=\bigcup_{n \geq 1} \widetilde{P}_{k}^{(n)}
$$

Let $C_{\emptyset}:=\left\{f \in \widetilde{P}_{k} \mid \operatorname{dom}(f)=\emptyset\right\}$.

The $n$-ary function $e_{i}^{(n)}$ defined by $e_{i}^{(n)}\left(x_{1}, \ldots, x_{n}\right):=$ $x_{i}$ with $i \in\{1, \ldots, n\}$ is called projection onto the $i$-th coordinate. Let $J_{k}:=\left\{e_{i}^{(n)} \mid n \in \mathbb{N}, 1 \leq i \leq n\right\}$ be the set of all projections.

Let $f\left[g_{1}, \ldots, g_{n}\right] \in \widetilde{P}_{k}^{(m)}$ be the composition as given in [2] with $f \in \widetilde{P}_{k}^{(n)}$ and $g_{1}, \ldots, g_{n} \in \widetilde{P}_{k}^{(m)}$, i.e., $x \in$ $\operatorname{dom}\left(f\left[g_{1}, \ldots, g_{n}\right]\right)$ iff

$$
\left(x \in \bigcap_{i=1}^{n} \operatorname{dom}\left(g_{i}\right)\right) \wedge\left(g_{1}(x), \ldots, g_{n}(x)\right) \in \operatorname{dom}(f)
$$

and

$$
f\left[g_{1}, \ldots, g_{n}\right](x):=f\left(g_{1}(x), \ldots, g_{n}(x)\right)
$$

for all $x \in \operatorname{dom}\left(f\left[g_{1}, \ldots, g_{n}\right]\right)$.

A partial clone (clone) on $E_{k}$ is a composition closed subset of $\widetilde{P}_{k}\left(P_{k}\right)$ containing $J_{k}$. Relations are useful to describe the clones of $\widetilde{P}_{k}$. We often write the elements of relations as columns and a relation can then be given as a matrix. For example the relation $\varrho=$ $\{(0,1,2),(1,2,0),(3,4,5),(2,3,1)\}$ can also be written as

$$
\varrho=\left(\begin{array}{llll}
0 & 1 & 3 & 2 \\
1 & 2 & 4 & 3 \\
2 & 0 & 5 & 1
\end{array}\right) .
$$

Let a matrix be given by $C=\left(c_{i j}\right)_{h \times n}$. Then $c_{i *}$ are the rows of the matrix with $i \in\{1, \ldots, h\}$, i.e., $c_{i *}=$ $\left(c_{i 1}, c_{i 2}, \ldots, c_{i n}\right)$, and $c_{* j}$ are the columns of the matrix with $j \in\{1, \ldots, n\}$, i.e., $c_{* j}=\left(c_{1 j}, c_{2 j}, \ldots, c_{h j}\right)^{\mathrm{T}}$.

Let $\mathcal{R}_{k}^{(h)}$ be the set of all $h$-ary relations on $E_{k}$ and $\mathcal{R}_{k}:=\bigcup_{h \geq 1} \mathcal{R}_{k}^{(h)}$.

An $n$-ary function $f^{(n)} \in \widetilde{P}_{k}$ preserves an $h$-ary relation $\varrho^{(h)} \in \mathcal{R}_{k}$ iff for all $c_{* 1}, c_{* 2}, \ldots, c_{* n} \in \varrho$ with $c_{1 *}, \ldots, c_{h *} \in \operatorname{dom}(f)$ holds

$$
f\left(c_{* 1}, \ldots, c_{* n}\right):=\left(\begin{array}{c}
f\left(c_{11}, \ldots, c_{1 n}\right) \\
f\left(c_{21}, \ldots, c_{2 n}\right) \\
\vdots \\
f\left(c_{h 1}, \ldots, c_{h n}\right)
\end{array}\right) \in \varrho .
$$

Let $\mathrm{pPOL}_{k} \varrho$ be the set of all functions $f \in \widetilde{P}_{k}$ which preserve the relation $\varrho \in \mathcal{R}_{k}$.

Let $f \in \widetilde{P}_{k}^{(1)}$ be a unary function. Define $f^{0}:=e_{1}^{(1)}$ and $f^{n}(x):=f\left(f^{n-1}(x)\right)$ for all $n \geq 1$.

For each $m \in \mathbb{N}$ let $\eta_{m}:=(0,1, \ldots, m-1)^{\mathrm{T}}$.

Let $\omega(v)$ be the set of entries of $v=\left(v_{1}, \ldots, v_{h}\right) \in E_{k}^{h}$, i.e., $\omega(v)=\omega\left(\left(v_{1}, \ldots, v_{h}\right)\right):=\left\{v_{1}, \ldots, v_{h}\right\}$. Additionally let $\omega(\varrho)=\bigcup_{v \in \varrho} \omega(v)$. 


\section{Theorem of Haddad and Rosenberg}

Definition 1. Let for all $h$ with $1 \leq h \leq k$

$$
\begin{aligned}
& \varrho_{1}:=\left\{(a, a, b, b),(a, b, a, b) \mid a, b \in E_{k}\right\}, \\
& \varrho_{2}:=\left\{(a, a, b, b),(a, b, a, b),(a, b, b, a) \mid a, b \in E_{k}\right\}, \\
& \iota_{k}^{h}:=\left\{\left(x_{1}, \ldots, x_{h}\right) \in E_{k}^{h}||\left\{x_{1}, \ldots, x_{h}\right\} \mid \leq h-1\right\} .
\end{aligned}
$$

Definition 2. Let $\varepsilon$ be an arbitrary equivalence relation on $E_{h}$. Define $\delta_{k, \varepsilon}^{(h)}:=$ $\left\{\left(a_{0}, \ldots, a_{h-1}\right) \in E_{k}^{h} \mid(i, j) \in \varepsilon \Longrightarrow a_{i}=a_{j}\right\}$. If $h$ or $k$ can be deduced from the context we just write $\delta_{\varepsilon}$ or $\delta_{\varepsilon}^{(h)}$ or $\delta_{k, \varepsilon}$. If the relation $\varepsilon$ is given by the nonsingular equivalence classes $\varepsilon_{1}, \ldots, \varepsilon_{r}$ then we write $\delta_{k ; \varepsilon_{1}, \ldots, \varepsilon_{r}}^{(h)}$ or $\delta_{\varepsilon_{1}, \ldots, \varepsilon_{r}}$ instead of $\delta_{k, \varepsilon}^{(h)}$. For example $\delta_{k ; E_{h}}^{(h)}=\left\{(x, x, \ldots, x) \in E_{k}^{h} \mid x \in E_{k}\right\}$.

Definition 3. Let $\varrho^{(h)} \subseteq E_{k}^{h}$. Then we write $\sigma(\varrho):=\varrho \backslash$ $\iota_{k}^{h}$ and $\delta(\varrho):=\varrho \cap \iota_{k}^{h}=\varrho \backslash \sigma(\varrho)$. If $\delta=\delta_{\varepsilon}$ for some equivalence relation $\varepsilon$ then we write $\varepsilon(\varrho):=\varepsilon$.

Definition 4. Let $\varrho^{(h)} \subseteq E_{k}^{h}$. Then $\varrho$ is

- areflexive, if $h \geq 2$ and $\delta(\varrho)=\emptyset$, i.e., for each $\left(x_{1}, \ldots, x_{h}\right) \in \varrho$ we have $x_{i} \neq x_{j}$ for all $1 \leq i<$ $j \leq h$.

- quasi-diagonal, if $\sigma(\varrho)$ is a non-empty areflexive relation, $\delta(\varrho)=\delta_{\varepsilon}$ with $\varepsilon \neq \iota_{h}^{2}$ an equivalence relation.

Definition 5. Let $\varrho^{(h)} \subseteq E_{k}^{h}, \sigma:=\sigma(\varrho)$ and $\delta:=\delta(\varrho)$.

If $r=\left(r_{0}, r_{1}, \ldots, r_{n-1}\right) \in E_{k}^{n}$ is a tuple and $\pi \in S_{n}$ then we write $r^{[\pi]}:=\left(r_{\pi(0)}, r_{\pi(1)}, \ldots, r_{\pi(n-1)}\right)$. Let $\Gamma_{\sigma}:=\left\{\pi \in S_{h} \mid \sigma \cap \sigma^{[\pi]} \neq \emptyset\right\}$, where $S_{h}$ is the set of all permutations on $E_{h}$ and $\sigma^{[\pi]}:=\left\{s^{[\pi]} \mid s \in \sigma\right\}$.

The model of $\varrho$ is the $h$-ary relation $M(\varrho):=$ $\left\{\eta_{h}^{[\pi]} \mid \pi \in \Gamma_{\sigma}\right\} \cup\left(\delta \cap E_{h}^{h}\right)$ on $E_{h}$. hold:

1. $\varrho \neq E_{k}^{h}, \varrho \neq \emptyset$,

2. (a) $\varrho$ is a unary relation, i.e., $h=1$, or

(b) $\varrho$ is areflexive with $2 \leq h \leq k$, or

(c) $\varrho$ is quasi-diagonal with $2 \leq h \leq k$, or

(d) $\delta=\iota_{k}^{h}$ with $3 \leq h \leq k$, or

(e) $\delta=\varrho_{i}$ with $i \in\{1,2\}$ (see Definition 1) and $h=4$,

3. $r^{[\pi]} \in \sigma$ for all $r \in \sigma$ and all $\pi \in \Gamma_{\sigma}$,

4. for every $\sigma^{\prime}$ with $\emptyset \neq \sigma^{\prime} \subseteq \sigma$ there is a relational homomorphism $\varphi: E_{k} \rightarrow E_{h}$ from $\sigma^{\prime}$ to $M(\varrho)$, such that $\varphi(r)=\eta_{h}$ for some $r \in \sigma^{\prime}$, i.e., $\left(\varphi\left(r_{0}\right), \ldots, \varphi\left(r_{h-1}\right)\right)=(0, \ldots, h-1)$ for some $r=$ $\left(r_{0}, \ldots, r_{h-1}\right) \in \sigma^{\prime}$
5. (a) if $\delta=\iota_{k}^{h}$ and $h \geq 3$ then $\Gamma_{\sigma}=S_{h}$,

(b) if $\delta=\varrho_{1}$ then $\Gamma_{\sigma}=\langle(0231),(12)\rangle\left(\Gamma_{\sigma}\right.$ is the permutation group generated by the cycles (0231) and (12)),

(c) if $\delta=\varrho_{2}$ then $\Gamma_{\sigma}=S_{4}$.

Let $\widetilde{\mathcal{R}}_{k}^{\max }$ be the set of all coherent relations with $\mathrm{pPOL}_{k} \varrho \neq \mathrm{pPOL}_{k} \chi$ for all $\varrho, \chi \in \widetilde{\mathcal{R}}_{k}^{\max }$ and $\varrho \neq \chi$. Let

$$
p \mathscr{M}_{k}:=\left\{P_{k} \cup C_{\emptyset}\right\} \cup\left\{\mathrm{pPOL}_{k} \varrho \mid \varrho \in \widetilde{\mathcal{R}}_{k}^{\max }\right\} .
$$

Theorem 6 (of Haddad and Rosenberg; [3, 4]). Let $k \geq 2$. For each $A \subset \widetilde{P}_{k}$ with $A=[A]_{\mathrm{P}}$ there is a maximal partial clone $M_{A}$ with $A \subseteq M_{A}$. A clone $M$ is a maximal partial clone of $\widetilde{P}_{k}$ if and only if $M \in p \mathscr{M}_{k}$, i.e., $p \mathscr{M}_{k}$ is the set of all maximal partial clones of $\widetilde{P}_{k}$.

Theorem 7 (Completeness criterion for $\widetilde{P}_{k} ;$ [4]). Let $C \subseteq$ $\widetilde{P}_{k}$. Then $[C]_{\mathrm{P}}=\widetilde{P}_{k}$ if and only if $C \nsubseteq M$ for all $M \in$ $p \mathscr{M}_{k}$.

Definition 8. The set of coherent relations $\widetilde{\mathcal{R}}_{k}^{\max }$ can be divided into the following sets:

$$
\begin{aligned}
\mathcal{U} & :=\left\{\chi^{(\mu)} \in \widetilde{\mathcal{R}}_{k}^{\max } \mid \mu=1\right\}, \\
\mathcal{A} & :=\left\{\chi^{(\mu)} \in \widetilde{\mathcal{R}}_{k}^{\max } \mid \mu \geq 2 \wedge \chi \text { is areflexive }\right\}, \\
\mathcal{Q} & :=\left\{\chi^{(\mu)} \in \widetilde{\mathcal{R}}_{k}^{\max } \mid \mu \geq 2 \wedge \chi \text { is quasi-diagonal }\right\}, \\
\mathcal{S} & :=\left\{\chi^{(\mu)} \in \widetilde{\mathcal{R}}_{k}^{\max } \mid \mu \geq 3 \wedge \delta(\chi)=\iota_{k}^{\mu}\right\}, \\
\mathcal{L} & :=\left\{\chi^{(\mu)} \in \widetilde{\mathcal{R}}_{k}^{\max } \mid \mu=4 \wedge \delta(\chi) \in\left\{\varrho_{1}, \varrho_{2}\right\}\right\} .
\end{aligned}
$$

\section{Number of maximal partial clones and maximal clones}

Definition 9. Let $\varrho^{(h)} \in \widetilde{\mathcal{R}}_{k}^{\max }$

Define the relation-class $\operatorname{class}(\varrho)$ by

$$
\begin{aligned}
& \operatorname{class}(\varrho):=\left\{\left\{(g(v))^{[\pi]} \mid v \in \varrho\right\} \mid g \in S_{k}, \pi \in S_{h}\right\} . \\
& \text { Let } p \mathscr{M}_{k}^{\mathrm{C}}:=\left\{\operatorname{class}(\varrho) \mid \varrho \in \widetilde{\mathcal{R}}_{k}^{\max }\right\} .
\end{aligned}
$$

For the number of maximal partial clones $\left|p \mathscr{M}_{k}\right|$ in $k$ valued logic with $k \in\{2,3,4,5,6\}$ see Table 3 . These are compared to the number of maximal (total) clones $\left|\mathscr{M}_{k}\right|$ as given in [7]. See Tables 1 and 2 for more detailed information about which types of relations contribute to the total number of coherent relations. The relations are split by arity which is shown in the second row of the header of the Tables. One can see that the ternary quasi-diagonal and areflexive relations contribute the biggest part to the number of maximal partial clones for $k \geq 4$. Especially the ternary relations $\varrho \in \mathcal{Q}$ with $\delta(\varrho)=\delta_{\{0,1\}}^{3}$ contribute 292440 for 
$k=5$ and 5008453443 for $k=6$. That means these relations alone determine the magnitude of $\left|p \mathscr{M}_{k}\right|$, at least for these cases.

These numbers were found by a computer program which is described in [11]. The program is written in Haskell, is single-threaded and took about 52 hours on a SunFire V490 to compute all coherent relations for $k=6$. The numbers found coincide with previous results; see [1] for $k=2$, [6] and [8] (independent from each other) for $k=3$, and [5] with corrections in [10] for $k=4$.

The size of $p \mathscr{M}_{k}^{\mathrm{C}}$ seems to be related to $\left|p \mathscr{M}_{k}\right|$ in an interesting way as can be seen in Table 4 . It seems reasonably to assume that

$$
\left|p \mathscr{M}_{k}\right| \sim k ! \cdot\left|p \mathscr{M}_{k}^{\mathrm{C}}\right|
$$

for $k \geq 6$. Because $\operatorname{pPOL}_{k} \varrho=\operatorname{pPOL}_{k} \varrho^{[\pi]}$ for any $\varrho^{(h)}$ and $\pi \in S_{h}$, there are at most $\left|S_{k}\right|=k$ ! different partial clones for every class $(\varrho)$. Thus clearly $\left|p \mathscr{M}_{k}\right| \leq k ! \cdot\left|p \mathscr{M}_{k}^{\mathrm{C}}\right|$.

Thinking about automatic checking for completeness of sets $C \subset \widetilde{P}_{k}$ the following idea might reduce the memory size needed for these checks.

Definition 10. Let $f^{(n)} \in \widetilde{P}_{k}$ and $g \in S_{k}$. Then define $F^{(n)}:=f^{g}$ by

$\operatorname{dom}(F):=\left\{\left(g^{-1}\left(x_{1}\right), \ldots, g^{-1}\left(x_{n}\right)\right) \mid\left(x_{1}, \ldots, x_{n}\right) \in \operatorname{dom} f\right\}$

and

$$
f^{g}\left(y_{1}, \ldots, y_{n}\right):=g^{-1}\left(f\left(g\left(y_{1}\right), \ldots, g\left(y_{n}\right)\right)\right)
$$

for all $\left(y_{1}, \ldots, y_{n}\right) \in \operatorname{dom}(F)$.

For $U \leq S_{k}$ let $f^{U}:=\left\{f^{g} \mid g \in U\right\}$ and $f^{\star}:=f^{S_{k}}$. For $C \subseteq \widetilde{P}_{k}$ let $C^{U}:=\left\{f^{U} \mid f \in C\right\}$ and $C^{\star}:=C^{S_{k}}$.

Theorem 11. Let $C \subseteq \widetilde{P}_{k}$ and $T \subseteq \widetilde{\mathcal{R}}_{k}^{\max }$ with

$$
\widetilde{\mathcal{R}}_{k}^{\max } \subseteq \bigcup_{\varrho \in T} \operatorname{class}(\varrho)
$$

Then $[C]_{\mathrm{P}}=\widetilde{P}_{k}$ if and only if

- $C \nsubseteq P_{k} \cup C_{\infty}$, and

- $C^{g} \nsubseteq \mathrm{pPOL}_{k} \varrho$ for all $\varrho \in T$ and all $g \in S_{k}$.

Proof. Let $M \in p \mathscr{M}_{k} \backslash\left\{P_{k} \cup C_{\infty}\right\}$. Then there is some $\chi^{(h)} \in \widetilde{\mathcal{R}}_{k}^{\max }$ with $M=\mathrm{pPOL}_{k} \chi$ by Theorem 6 , and by assumption there is some $\varrho \in T$ with $\chi \in \operatorname{class}(\varrho)$. Thus we have some $g \in S_{k}$ and $\pi \in S_{h}$ with

$$
\chi=\left\{g(v)^{[\pi]} \mid v \in \varrho\right\},
$$

where we can assume w.l.o.g. $\pi=\mathrm{id}$. We have $C^{g} \notin$ $\mathrm{pPOL}_{k} \varrho$, thus there is some $f^{(n)} \in C$ and $v_{1}, \ldots, v_{n} \in \varrho$ with

$$
f^{g}\left(v_{1}, \ldots, v_{n}\right):=w \in E_{k}^{h} \backslash \varrho .
$$

Thus

$$
f\left(g\left(v_{1}\right), \ldots, g\left(v_{n}\right)\right)=g(w) \in g\left(E_{k}^{h}\right) \backslash g(\varrho)=E_{k}^{h} \backslash \chi
$$

with $g\left(v_{i}\right) \in g(\varrho)=\chi$ for all $i \in\{1, \ldots, n\}$. That means $f \notin \mathrm{pPOL}_{k} \chi=M$.

Thus $C \nsubseteq M$ for all $M \in p \mathscr{M}_{k}$ and by Theorem 7 we have $[C]_{\mathrm{P}}=\widetilde{P}_{k}$.

Example 12. Let $k=6$ and we want to check if $C=$ $\left\{f_{1}, \ldots, f_{l}\right\}$ is complete, i.e., $[C]_{\mathrm{P}}=\widetilde{P}_{k}$. We assume the following setting.

- Every tuple in the domain of an $f \in C$ needs $t=10$ Bytes on average to store.

- There are less than 1000 tuples in all domains combined, i.e.,

$$
d:=\sum_{f \in C}|\operatorname{dom} f|<1000 .
$$

Because there exist binary partial Sheffer functions for $k=6$, i.e., with less than 36 tuples in the domain, the restriction to 1000 tuples is not too restrictive.

- Every coherent relation takes about $r=10$ Bytes to store in a convenient format for testing preservation of relations.

If we use the direct approach of storing all coherent relations in memory, then we need at least $r \cdot\left|p \mathscr{M}_{k}\right|=$ $10 \cdot 5242621816$ Bytes, approximately 50 Gigabytes.

If we use Theorem 11 instead, then we need about $t \cdot d$. $k !+r \cdot\left|p \mathscr{M}_{k}^{\mathrm{C}}\right|<10 \cdot 1000 \cdot 720+10 \cdot 7322017=80420170$ Bytes, approximately 80 Megabytes.

As $\left|p \mathscr{M}_{k}\right| \approx k !\left|p \mathscr{M}_{k}^{\mathrm{C}}\right|$ for $k=6$ we have to make about the same number of tests in either case. Furthermore it is fast to generate the set $C^{g}$ for all $g \in S_{k}$. Thus the use of Theorem 11 reduces the memory consumption considerably while leaving the number of tests nearly constant.

For $k=5$ we have $r \cdot\left|p \mathscr{M}_{k}\right|=3257220$ and $t \cdot d \cdot k !+$ $r \cdot\left|p \mathscr{M}_{k}\right|<1232870$ Bytes for the different approaches, respectively. This is not so important with the todays memory sizes, and we would make about $20 \%$ more tests with the second approach compared to the first. Thus the second approach would be worse for $k=5$ in the given scenario.

Unfortunately even the number of relation classes $\left|p \mathscr{M}_{k}^{\mathrm{C}}\right|$ will propably grow very fast since $\left|p \mathscr{\mathscr { M }}_{k}^{\mathrm{C}}\right|>\left|\mathscr{M}_{k}\right|$ for all $k \leq 6$. Thus a list of coherent relations seems not practical for bigger $k$. Under these circumstances we try to use the description of coherent relations directly without generating all coherent relations. We cannot hope to find precise criteria for completeness but sufficient and necessary conditions for complete systems should help in practice. The next chapter states such conditions. 


\section{Other completeness criteria}

The number of coherent relations is quite large and it is computationally difficult to find all of them. Thus we give a list of coherent relations which are easy to enumerate and functions are easy to check for preservation against these relations. These give some necessary conditions for complete function systems.

Example 13. Let $C \subseteq \widetilde{P}_{k}$ with $[C]_{\mathrm{P}}=\widetilde{P}_{k}$. Then $C \nsubseteq$ $\operatorname{pPOL}_{k} \varrho$ for all $\varrho \in \mathcal{U} \cup\left\{\iota_{k}^{h} \mid 3 \leq h \leq k\right\}$.

If $\varrho \in \mathcal{U}$ then we can check if $C \subseteq \mathrm{pPOL}_{k} \varrho$ in at most $\sum_{f \in C}|\operatorname{dom} f|$ steps, because every tuple in $\operatorname{dom} f$ is independent of each other with regard to $\varrho$ in this case. Additionally, the set $\mathcal{U}=\left\{\chi \subset E_{k} \mid \chi \neq \emptyset\right\}$ can be enumerated fast. For $k=6$ with the setting from the example above there are at most $|\mathcal{U}| \cdot \sum_{f \in C}|\operatorname{dom} f|<2^{6} \cdot 1000=64000$ single tuple tests to be done. This is very small with respect to checking all coherent relations.

If $\varrho \in\left\{\iota_{k}^{h} \mid 3 \leq h \leq k\right\}$ then the test is easy, and most expensive if $C=\{f\}$ for some function $f \in \widetilde{P}_{k}$, so just assume this is the case. Just take any $h$ tuples $s_{1 *}, \ldots, s_{h *} \in$ $\operatorname{dom} f$ with $\left|\left\{f\left(s_{i *}\right) \mid i \in\{1, \ldots, h\}\right\}\right|=h$ and check that for every column $s_{* j}$ we have at most $h-1$ different entries, i.e. $\left|\left\{s_{i j} \mid i \in\{1, \ldots, h\}\right\}\right| \leq h-1$.

The next theorem gives a sufficient condition for completeness.

\section{Theorem 14. Let}

- $C:=\left\{f_{1}^{\left(h_{1}\right)}, \ldots, f_{l}^{\left(h_{l}\right)}\right\} \subseteq \widetilde{P}_{k}$,

- $\varphi_{1}, \ldots, \varphi_{m} \in S_{k}$,

- $U_{j}:=\left\{\varphi(v) \mid v \in U_{j-1}, \varphi \in\left\langle\varphi_{1}, \ldots, \varphi_{j}\right\rangle\right\}$ for $j \in$ $\{1, \ldots, m\}$ and $U_{0}:=\left\{\eta_{k}\right\}$.

Let

1. $\left\langle\varphi_{1}, \ldots, \varphi_{m}\right\rangle=S_{k}$,

2. for all $j \in\{1, \ldots, m\}$ there are $i \in\{1, \ldots, l\}$ and $v_{1}, \ldots, v_{h_{i}} \in U_{j-1}$ with $f_{i}\left(v_{1}, \ldots, v_{h_{i}}\right)=\varphi_{j}\left(\eta_{k}\right)$,

3. there are $i \in\{1, \ldots, l\}$ and $v_{1}, \ldots, v_{h_{i}} \in U_{m}$ with $f_{i}\left(v_{1}, \ldots, v_{h_{i}}\right) \in \delta_{\varepsilon}^{(k)}$ for some non-trivial equivalence relation $\varepsilon$ such that there is some $x \in E_{k}$ with $(x, y) \notin \varepsilon$ for all $y \in E_{k} \backslash\{x\}$, i.e., $\varepsilon$ has a singular equivalence class,

4. for all $\chi \in\left\{\iota_{k}^{h} \mid h \in\{3, \ldots, k\}\right\} \cup\left\{\varrho_{1}, \varrho_{2}\right\}$ there is some $f \in C$ with $f \notin \mathrm{pPOL}_{k} \chi$, and

5. there is some $f \in C$ with $f \notin P_{k} \cup C_{\infty}$.

Then $[C]_{\mathrm{P}}=\widetilde{P}_{k}$.
Proof. Assume $[C]_{\mathrm{P}} \neq \widetilde{P}_{k}$. Then there is some $M \in p \mathscr{M}_{k}$ with $C \subseteq M$. Because there is some $f \in C$ with $f \notin P_{k} \cup$ $C_{\infty}$ we know that there is some coherent relation $\varrho^{(h)} \in$ $\widetilde{\mathcal{R}}_{k}^{\max }$ with $M=\mathrm{pPOL}_{k} \varrho$.

- We first consider $\sigma(\varrho)=\emptyset$. Then $\varrho \in\left\{\iota_{k}^{h} \mid h \in\right.$ $\{3, \ldots, k\}\} \cup\left\{\varrho_{1}, \varrho_{2}\right\}$ and thus there is some $f \in C$ with $f \notin \mathrm{pPOL}_{k} \varrho$, i.e. $C \nsubseteq M$.

- Now we see that there is some $v \in \sigma(\varrho)$ and we can assume $v=\eta_{h}$. That means $\{v\}=\operatorname{pr}_{h} U_{0}:=$ $\operatorname{pr}_{0,1, \ldots, h-1} U_{0}$ and by (2) there is some function $f \in$ $C$ such that $\varphi_{1}(v)=f(v, \ldots, v) \in \varrho$ because $f \in$ $\mathrm{pPOL}_{k} \varrho$. Doing this repeatedly we get $\operatorname{pr}_{h} U_{1}=$ $\left\{\varphi(v) \mid \varphi \in\left\langle\varphi_{1}\right\rangle\right\} \subseteq \varrho$.

By iteration we get $\mathrm{pr}_{h} U_{2}, \mathrm{pr}_{h} U_{3}, \ldots, \mathrm{pr}_{h} U_{m} \subseteq \varrho$. Thus $\sigma\left(E_{k}^{h}\right) \subseteq \sigma(\varrho)$ because $\left\langle\varphi_{1}, \ldots, \varphi_{m}\right\rangle=S_{k}$ and therefore $U_{m}=\sigma\left(E_{k}^{k}\right)$. This implies that $G_{\sigma(\varrho)}=S_{h}$, i.e. $\varrho$ is totally-symmetric.

If $h=1$ then $\sigma\left(E_{k}^{h}\right)=E_{k}$ and thus $\varrho=E_{k}$, i.e. $\varrho \notin \widetilde{\mathcal{R}}_{k}^{\max }$, in contradiction to the assumption.

For $h \geq 2$ we know by $(3)$ that $\delta(\varrho) \neq \emptyset$. This means that $\delta(\varrho)=\iota_{k}^{2}$ because $\varrho$ is coherent. But then $\varrho=E_{k}^{2}$ in contradiction to the assumption that $\varrho$ is coherent.

For $h \geq 3$ we even know that $\delta(\varrho) \neq \delta_{\varepsilon}$ for all $\varepsilon$ without singleton class. Assume $\delta(\varrho)=\delta_{\varepsilon}$ and $\varepsilon$ has at least one singleton class. But then there is some $\pi \in$ $S_{h}$ with $\delta(\varrho)^{[\pi]} \neq \delta(\varrho)$ in contradiction to $\varrho$ coherent.

Thus $\delta(\varrho) \in\left\{\iota_{k}^{h}, \varrho_{1}, \varrho_{2}\right\}$. If $h=4$ then $\delta(\varrho) \neq \varrho_{i}$ for $i \in\{1,2\}$ because of (3), i.e., $f_{i}\left(v_{1}, \ldots, v_{h_{i}}\right)=$ $(x, x, y, z) \in \delta\left(E_{k}^{4}\right) \backslash \varrho_{i}$. Thus we see that $\delta(\varrho)=\iota_{k}^{h}=$ $\delta\left(E_{k}^{h}\right)$. But then $\varrho=\sigma(\varrho) \cup \delta(\varrho)=E_{k}^{h}$ contradicting $\varrho$ coherent.

Thus we can conclude that $C \nsubseteq M$ for all $M \in p \mathscr{M}_{k}$ and by Theorem 7 we get $[C]_{\mathrm{P}}=\widetilde{P}_{k}$.

\section{Conclusion}

We have given the number of maximal partial clones up to $k=6$ which forced us to think about different ways for checking for completeness of sets of partial functions. It might be interesting to investigate this further and find different approaches. There are still some open problems such as the following:

- What are good candidate sets $T$ for Theorem 11 ?

- Is there a formula for the number of maximal partial clones like the one for the number of maximal total clones? See [9]. 


\section{Acknowledgment}

The author would like to thank his advisor Dietlinde Lau for the wonderful support.

\section{References}

[1] R. Freivald. Completeness criteria for partial functions of the algebra of logic and many-valued logics, english translation. Dokl. Akad. Nauk. SSSR 167, 6:1249-1250, 1966.

[2] L. Haddad and D. Lau. Some criteria for partial Sheffer functions in $k$-valued logic. Mult.-Valued Logic and Soft Computing, 13(4-6):415-446, 2007.

[3] L. Haddad and I. Rosenberg. Maximal partial clones determined by the areflexive relations. Discrete Appl. Math., 24(1-3):133-143, 1989.

[4] L. Haddad and I. Rosenberg. Completeness theory for finite partial algebras. Algebra Univers., 29(3):378-401, 1992.

[5] L. Haddad and G. Simons. Maximal partial clones of 4-valued logic. Mult.-Valued Logic and Soft Computing, 8(4):531-562, 2002.

[6] D. Lau. Eigenschaften gewisser abgeschlossener Klassen in Postschen Algebren. Dissertation a, Universität Rostock, 1977.

[7] D. Lau. Function algebras on finite sets. A basic course on many-valued logic and clone theory. Springer Monographs in Mathematics. Berlin: Springer. xiv, 668 p., 2006.

[8] B. Romov. Maximal subalgebras of algebras of partial multivalued logic functions. Cybernetics, 16:31-41, 1980. translation of Kibernetica 1980, No. 1, 28-36 (1980).

[9] I. Rosenberg. The number of maximal closed classes in the set of functions over a finite domain. Journal of Combinatorial Theory, Series A, 14(1):1 - 7, 1973.

[10] K. Schölzel. The minimal covering of maximal partial clones in 4-valued logic. In 39th International Symposium on Multiple-Valued Logic, pages 126-131, May 2009.

[11] K. Schölzel. Number of maximal partial clones. MultipleValued Logic and Soft Computing, 2009. submitted. 


\begin{tabular}{c|c|cccc|cc|c}
$k$ & $\left|p \mathscr{M}_{k}\right|$ & & $|\mathcal{S}|$ & & $|\mathcal{L}|$ & $P_{k} \cup C_{\emptyset}$ \\
& & 3 & 4 & 5 & 6 & & & \\
\hline 2 & 8 & & & & 1 & 1 & 1 \\
3 & 58 & 1 & & & 1 & 1 & 1 \\
4 & 1102 & 15 & 1 & & 4 & 2 & 1 \\
5 & 325722 & 1023 & 31 & 1 & & 46 & 16 & 1 \\
6 & 5242621816 & 1048575 & 32767 & 63 & 1 & 4141 & 786 & 1
\end{tabular}

Table 1. Number of maximal partial clones I

\begin{tabular}{c|cccccc}
$k$ & \multicolumn{7}{|c}{$|\mathcal{Q} \cup \mathcal{A}|$} \\
& 1 & 2 & 3 & 4 & 5 & 6 \\
\hline 2 & 2 & 3 & & & & \\
3 & 6 & 30 & 18 & & & \\
4 & 14 & 416 & 505 & 144 & & \\
5 & 30 & 16457 & 295080 & 11945 & 1092 & \\
6 & 62 & 1934514 & 5008589703 & 230676900 & 319722 & 14581
\end{tabular}

Table 2. Number of maximal partial clones II

\begin{tabular}{r|r|r}
$k$ & $\left|\mathscr{M}_{k}\right|$ & $\left|p \mathscr{M}_{k}\right|$ \\
\hline 2 & 5 & 8 \\
3 & 18 & 58 \\
4 & 82 & 1102 \\
5 & 643 & 325722 \\
6 & 15182 & 5242621816 \\
7 & 7848984 & $?$ \\
8 & 549761933169 & $?$
\end{tabular}

Table 3. Number of maximal (partial) clones

\begin{tabular}{c|c|c|c|c|c}
$k$ & $\left|p \mathscr{M}_{k}\right|$ & $\left|p \mathscr{M}_{k}^{\mathrm{C}}\right|$ & $\frac{\left|p \mathscr{M}_{k}\right|}{\left|p \mathscr{M}_{k}^{\mathrm{C}}\right|}$ & $k !$ & $\frac{\left|p \mathscr{M}_{k}\right|}{\left|p \mathscr{M}_{k}^{\mathrm{C}}\right| \cdot k !}$ \\
\hline 2 & 8 & 7 & 1.14 & 2 & 0.57 \\
3 & 58 & 26 & 2.23 & 6 & 0.37 \\
4 & 1102 & 138 & 7.99 & 24 & 0.33 \\
5 & 325722 & 3287 & 99.1 & 120 & 0.82 \\
6 & 5242621816 & 7322017 & 716 & 720 & 0.99
\end{tabular}

Table 4. Size of $p \mathscr{M}_{k}^{\mathrm{C}}$ in comparison to $p \mathscr{M}_{k}$ 